\title{
Management of ulcerated finger pad tophi
}

\author{
Sudipta Bera ${ }^{1}$, Shobhit Sharma ${ }^{1}$, Vivek Gupta ${ }^{2}$, Durgesh Kumar Pal ${ }^{1}$ \\ ${ }^{1}$ Department of Plastic Surgery, Shri Ram Murti Smarak Institute of Medical Sciences, Bareilly, Uttar Pradesh, India \\ ${ }^{2}$ Department of Plastic and Reconstructive Surgery, Sir Ganga Ram Hospital, New Delhi, India
}

\begin{abstract}
Finger pad tophi are an unusual but not very rare presentation of uncontrolled gout. Unlike tophaceous lesions of other locations, finger pad tophi are often difficult to diagnose at presentation due to resemblance to a variety of nodular lesions, coexistence with other connective tissue disorders and sometimes occurrence in asymptomatic hyperuricemia. They also pose a therapeutic dilemma due to a lack of clear treatment guidelines. Several case reports of finger pad tophi have been published before, focusing mainly on diagnosis and medical management. We discuss here a case of thumb pad tophi as primary presentation of undiagnosed asymptomatic hyperuricemia and treated surgically with excision and full-thickness skin graft along with urate-lowering therapy. This article highlights the diagnosis and treatment of finger pad tophi. We also discuss the surgical management of tophaceous lesions of hands and digits.
\end{abstract}

Key words: surgical treatment, ulcerated, finger pad tophi, full-thickness skin graft.

\section{Introduction}

Tophi from uncontrolled gout are usually seen on extremities. The first metatarsophalangeal (MTP) joint is commonly affected by the tophaceous deposits. However, finger pad tophi are relatively uncommon. Unlike tophaceous lesion of other locations, finger pad tophi are often difficult to diagnose at presentation due to resemblance to a variety of nodular lesions, coexistence with other connective tissue disorders and sometimes occurrence in asymptomatic hyperuricemia [1-4].

They also pose a therapeutic dilemma due to a lack of clear treatment guidelines. Several case reports of finger pad tophi have been published before, focusing mainly on diagnosis and medical management [1-6].

Urate-lowering therapy (ULT) has been the mainstay of the management of tophaceous lesions and uncontrolled gout. Surgical intervention is reserved for tophi producing ulceration, disfigurement, compressive symptoms, and functional disability [7]. Though use of ULT has reduced the incidence of extensive and complicated tophaceous lesions, involvement of the finger pad requires prompt diagnosis and management for early functional recovery of the hand.
The aim of the article is to review the literature based on the problem described in the present case of thumb pad tophi as primary presentation of undiagnosed asymptomatic hyperuricemia and treated surgically with excision and full-thickness skin graft (FTSG) along with ULT. We also discuss the surgical management of such lesions.

\section{Material and methods}

The PubMed and Google Scholar databases were searched using key words such as "tophaceous and gout", and "tophi". The result was refined with "finger pad", "digit", "hand", and "surgical management". The case report and previous described case series involving tophi of the hand and digit and surgical management of such lesions were reviewed.

\section{Case report}

A 56-year-old male patient, a carpenter by profession, attended the outpatient department with a history of gradually increasing nodular lesion over the volar aspect of the left thumb for the past one year. The lump ulcerated with persistent whitish chalky discharge one month

Address for correspondence:

Sudipta Bera, Department of Plastic Surgery, Shri Ram Murti Smarak Institute of Medical Sciences, Bareilly - Nainital Rd, Bhoji Pura,

243202 Uttar Pradesh, India, e-mail: sudiptacnmc@gmail.com

Submitted: 14.02.2020; Accepted: 27.04.2020 
before the consultation and he was complaining of painful limitation of thumb movement during daily activities.

The patient had no history of joint pain but incidental hyperuricemia was detected a few years back. He was not taking any medicines regularly for this. He was free from any symptoms of gout at the presentation. On examination, a $3 \mathrm{~cm} \times 2 \mathrm{~cm}$, painful ulcerative nodular lesion on the palmar aspect of the left thumb over the interphalangeal (IP) joint was noted. A white chalky discharge from the ulcer was noted (Fig. 1).

Fine needle aspiration cytology (FNAC) and MRI were done to diagnose the nature and extent of the lesion. FNAC showed negatively birefringent needle shaped crystals with overlying hyperplastic, hyperkeratotic skin and excluded a malignant lesion. MRI revealed

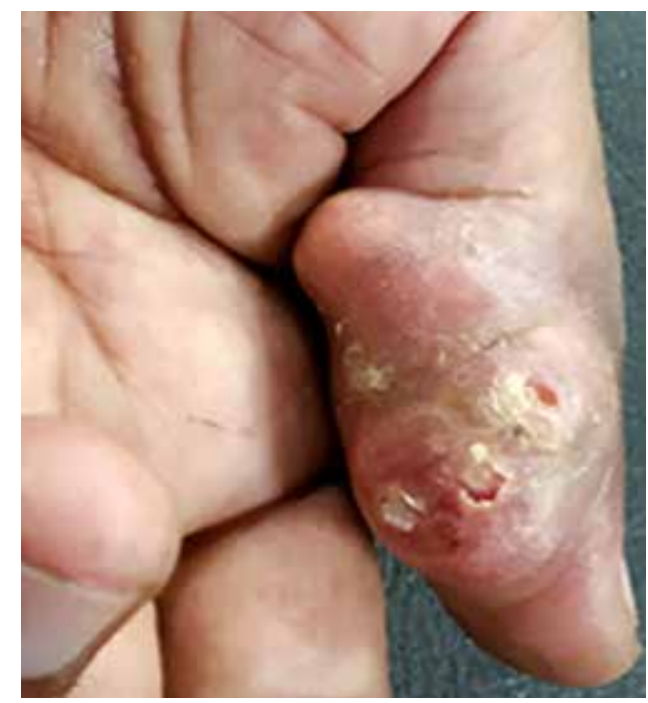

Fig. 1. Finger pad tophi with ulceration.

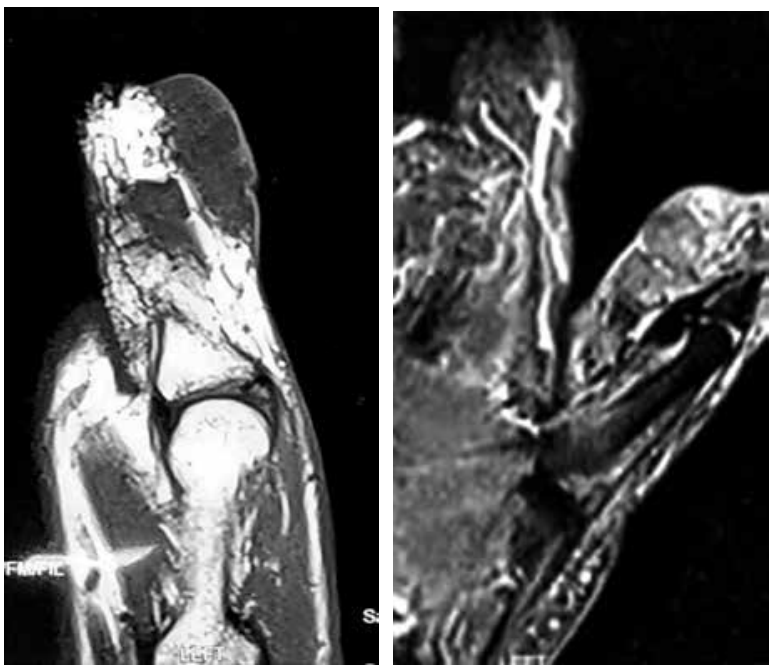

Fig. 2. T1- and T2-weighted MRI showing a hypointense lesion in the subcutaneous plane. a hypointense $\mathrm{T} 1$ and $\mathrm{T} 2$-weighted soft tissue lesion in a superficial plane to the bone and tendons (Fig. 2). The discharge culture was sterile. The serum uric acid measured $10.2 \mathrm{mg} / \mathrm{dl}$ and the patient was referred to the rheumatological department for further evaluation and to initiate medical management.

The lesion was operated on after initiating ULT as it was causing significant functional disability and pain. It was found to be infiltrated to skin and underlying tissue without a plane of separation. Hence it was excised with a scalpel blade as much as possible along with infiltrated skin and subcutaneous tissue superficial to the tendon plane. The wound bed was found to be studded with fine inseparable white granules and was irrigated with a warm saline jet. An FTSG was harvested from the medial aspect of the arm under local anesthesia. The graft was dressed with bolster dressing (Figs. 3A-3C).

The dressing was changed on the $10^{\text {th }}$ postoperative day and graft take was complete. The patient was kept on regular ULT with allopurinol starting from an initial dose of $100 \mathrm{mg} /$ day and physiotherapy. At the 6-month follow-up serum uric acid (UA) concentration was quite satisfactory $(6.2 \mathrm{mg} / \mathrm{dl})$. The goal was UA concentration below $6.0 \mathrm{mg} / \mathrm{dl}$ therefore eating habits were corrected. The goal was re-set to UA concentration up to $5.5 \mathrm{mg} / \mathrm{dl}$ and ULT therapy was titrated. No erosion of graft or contracture was noted after a 6-month follow-up. The patient resumed full use of his thumb and daily activity (Fig. 4).

\section{Results of literature overview}

21 cases of finger pad tophi from 12 different studies were identified. All cases were reported between 1983 and 2017 [1-6].

Tophi are deposits of uric acid (UA) crystals in the dermal and subdermal tissue, but also may occur in other body places including internal organs $[5,6]$. Finger pad tophi may present as nodular or macular lesions with a diagnostic dilemma. Diagnosis may be challenging as they closely resemble a variety of benign and malignant nodular lesions of the hand.

The differential diagnosis of such lesions includes: benign tumors such as tendon sheath fibroma, focal nodular synovitis, lipoma, lipofibromatous hamartoma, vascular malformations, neurofibroma, collagenous fibroma, glomus tumor, giant cell tumor (GCT) of the tendon sheath, chondroid tumor, and malignant tumors, such as fibrosarcoma of the tendon sheath, pleomorphic sarcoma, primary squamous cell carcinoma of the skin, and malignant bone chondrosarcoma.

They also include conditions resembling a mass lesion such as epidermal cyst, inflammatory mass aris- 

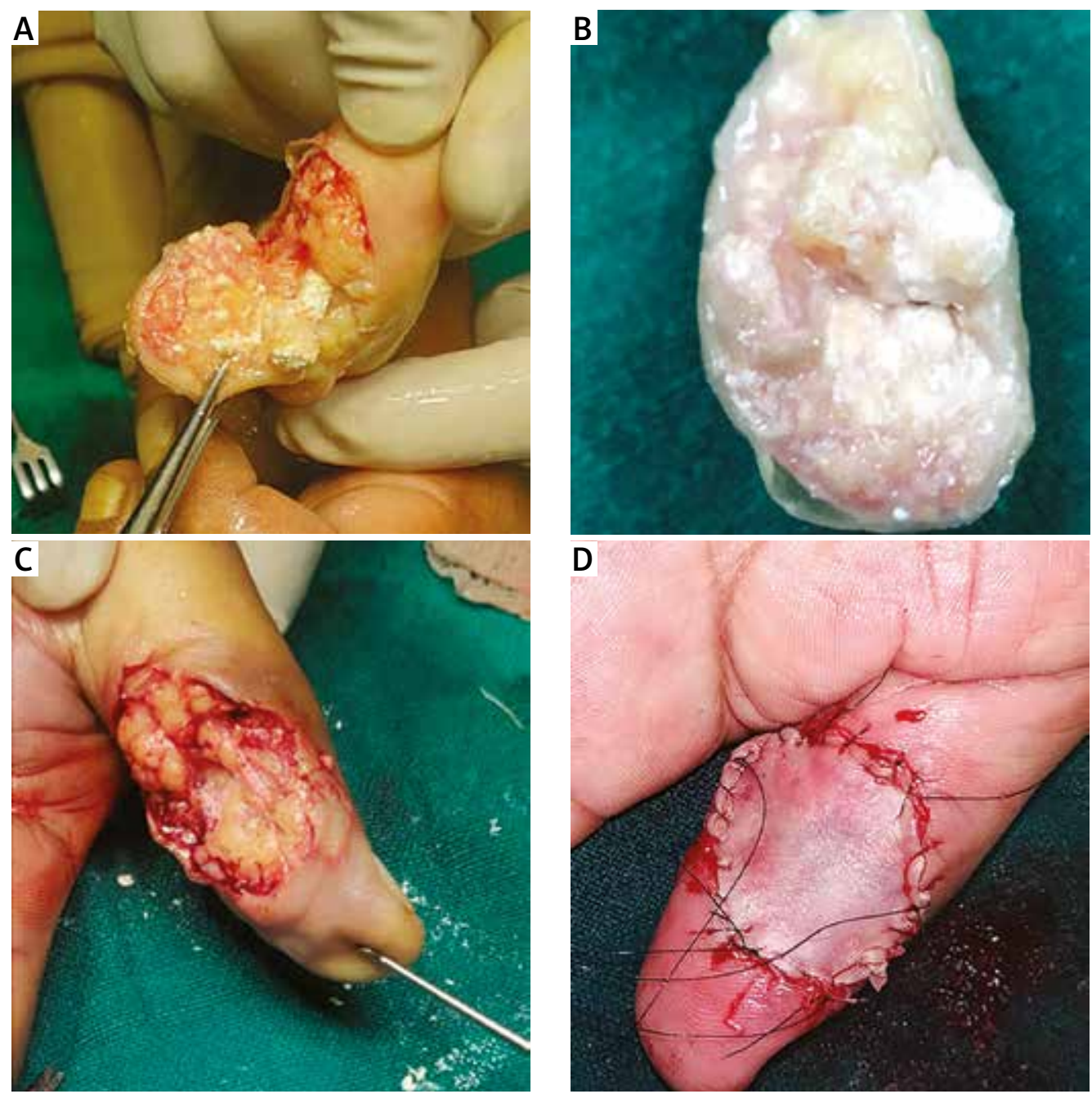

Fig. 3. Excision of the lesion. The lesion is noted firmly incorporated within the soft tissue (A); excised tissue (B); the remaining soft tissue bed studded with fine residual crystals (C); full-thickness skin graft coverage (D).

ing from local trauma and infections, tenosynovitis, etc. They closely resemble a nodular lesion arising from a connective tissue disorder such as rheumatoid nodules, and deposits such as skin calcification, chondrocalcinosis, xanthoma, and secondary deposits from kidney and lung cancers, etc. [2, 8, 9]. Atypical presentation with concomitant giant cell tumor of soft tissue or as a bullous lesion also has been reported $[10,11]$.

Diagnosis may be obscure as it may coexist with other connective tissue disorders. It may mimic a rheumatoid nodule, Heberden and Bouchard nodes, and calcinosis cutis when coexisting with rheumatoid arthritis, osteoarthritis or systemic sclerosis respectively. Finger pad tophi presenting with the Raynaud phenomenon and autoimmune disease such as systemic lupus erythematosus (SLE) has also been reported [4]. Moreover, they may present as the primary manifestation of asymptomatic or undiagnosed gout or in association with chronic renal failure (CRF) and the use of diuretics [1].

Fine needle aspiration cytology is essential for prompt diagnosis of such lesions. Histopathology of the lesions demonstrates characteristic negative birefringent needle-shaped crystals on polarized light. MRI indicates the size, morphology, and extent of the lesions. Hyperuricemia, characteristic chalky discharge, negative birefringent needle-shaped crystals on histopathology and MRI usually diagnose it sufficiently for management purposes [12-14].

Recently in some clinical centers dual energy computed tomography (DECT), which may also quickly confirm the presence of uric acid crystals using an appropriate protocol of this test, is available $[15,16]$.

Urate-lowering therapy is the mainstay of the management of tophaceous lesions. Tophi are common if 

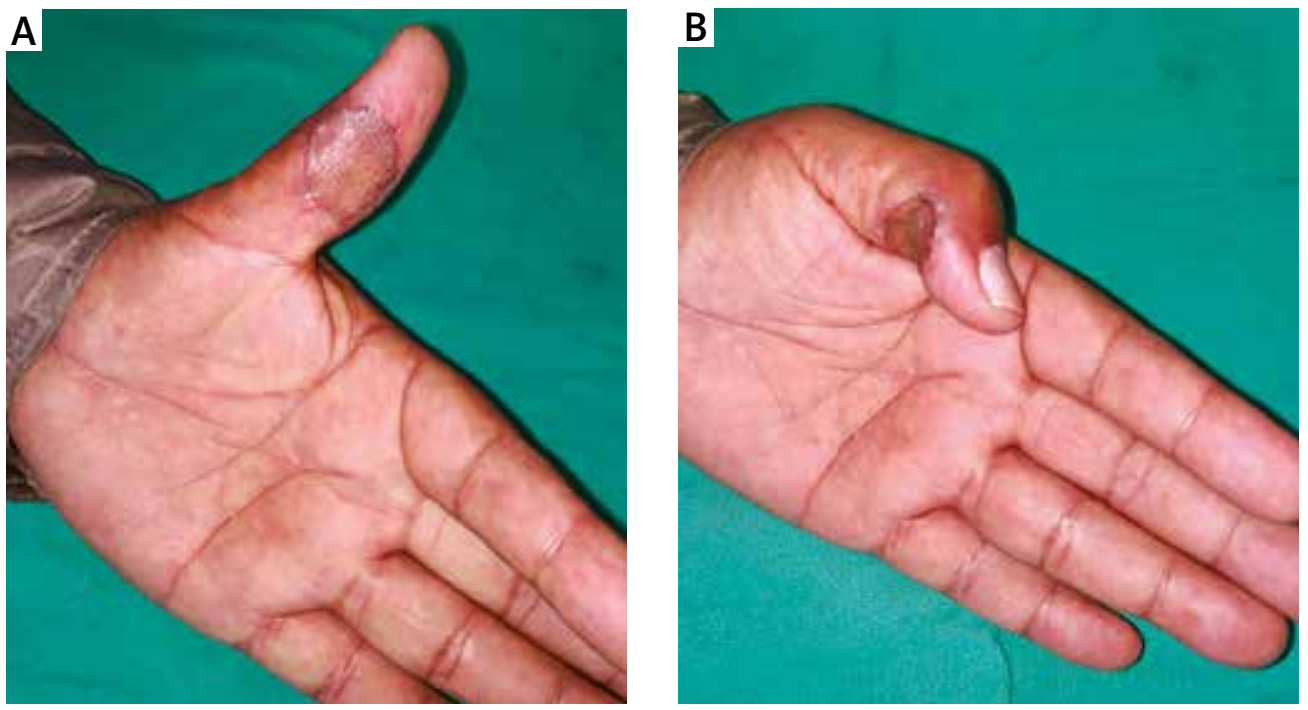

Fig. 4. Six month follow-up showing well-settled graft and full functional recovery.

UA remains as high as $10 \mathrm{mg} / \mathrm{dl}$. Keeping serum urate $<6 \mathrm{mg} / \mathrm{dl}$ not only prevents tophi but is also found to decrease the size of the tophi effectively. Allopurinol and febuxostat, a class of xanthine oxidase inhibitors (XOi), are presently recommended first-line drugs for single-agent therapy for chronic and tophaceous gout. Dose adjustment, use of drugs in combination or use of second-line drugs such as probenecid, lesinurad, or pegloticase may be required to tailor the medical therapy for optimum reduction of serum UA and tissue urate load in individual patients $[17,18]$.

However, the velocity of the reduction of the lesions is uncertain. For larger lesions, it is also time-consuming. Thus ULT is useful for a tophaceous lesion when the size of the tophi is small or involves a non-functional anatomical location. They may also have a role to clear the wound from residual crystals after debulking, which is otherwise difficult with surgery only.

Though in earlier days surgical intervention was preferred for a tophaceous lesion for cosmetic debulking, with the progress of ULT the need for surgical interventions for such lesions decreased unless they are complicated with painful skin ulceration, infection, discharging sinus, nerve compression, joint instability, and functional disability. They also may be considered along with ULA for locations that are functionally important and need quick removal of the lesion and lesions refractory to medical therapy.

Larmon et al. [7] and Straub et al. [19] discussed the indications of surgery in detail. To summarize, these indications are as listed below:

- Functional: joint instability, limitation of movement, etc.

- Symptomatic: pain, ulceration, infection, entrapment neuropathy, radiculopathy.
- Cosmetic: improve appearance particularly when they involve dorsum of the hand.

- Metabolic: persistent high serum uric acid (> $5.5 \mathrm{mg} / \mathrm{dl}$ ), refractory or unable to take medical therapy [7, 17, 20, 21].

The location, depth, and consistency of the lesions are determinant factors for the choice of surgery [7, 8, 17]. Surgery may be categorized as conservative or extensive.

\section{Conservative surgery}

The deposits are removed without sacrificing much of the involved structure. It includes debridement, debulking, irrigation, curettage, enucleation, shaving, endoscopic decompression, staged debridement, vacuum-assisted closure, etc. [8, 17, 20, 22-27].

These conservative approaches reduce the volume of deposit, pressure and obstructive symptoms and the chance of surface ulceration. The drawback of conservative debridement is delayed healing, wound-related complications from residual deposits and recurrence $[8,23]$. Thus it is most suitable for small, non-infiltrating and subcutaneous lesions.

\section{Extensive surgery}

It may be summarized as complex excision, tophectomy, tenosynovectomy, arthrodesis, arthroplasty, amputations, and reconstruction with skin grafts, flaps or bone grafts [ $8,17,18,25,26]$. These procedures radically remove the deposits along with the infiltrated anatomical structures by sharp dissection and simultaneous reconstructions of exposed parts. They are useful for deep infiltrating lesions, mainly on the dorsal hand and fingers and are indicated for tenosynovitis, joint destruction, bone erosion and compression neuropathy involving the deeper tissues. 
Complex excision removes the deposit as much as possible and improves pain and function. But it may require complex reconstruction, adding to further morbidity and complications such as joint stiffness, adhesions, graft loss, etc. [8, 20, 23]. Postoperative rehabilitation is essential for this purpose. Amputation may be considered for extensive damage to digits, devascularization or intractable pain. Simultaneous ULT is essential whatever the surgical approach is, as it reduces the residual deposits and recurrences.

Tophi on the dorsum of the hand may be large and grow rapidly as skin is loose and supple here. They may involve a deeper structure as there is a smaller amount of soft tissue barrier. In contrast, finger pad tophi spare deeper structure at presentation, probably due to the thick soft tissue cushion over bone and tendons. They are prone to cause surface ulceration as they tend to grow superficially and are subjected to repeated friction during daily activities. Due to skin infiltration, it is difficult to remove the deposit without sacrificing the skin $[8,22,26]$.

As finger pad tophi restrict essential use of the hand, they may be best managed with surgical intervention along with timely initiation of medical management to hasten functional recovery.

The present article focuses on full-thickness skin graft (FTSG) reconstruction after excision of ulcerated finger pad tophi. We observed that the clumps of deposits firmly infiltrated the skin and soft tissue in an inseparable manner. The loosely attached clumps and infiltrated skin could be removed sharply until the tendon plane was reached (Figs. $3 \mathrm{~A}$ and $3 \mathrm{~B}$ ).

The soft tissue bed after excision of large deposits remains studded with sparse fine crystals which could be cleared partially with irrigation of warm saline. Vascularity of the remaining pulp tissue bed remains well unless there is a coexisting connective tissue disorder (Fig. 3C). Immediate resurfacing with a split-thickness graft may risks graft loss from the underlying granular deposits and contracture later on [26], while coverage with a flap may be too extensive as tendons are usually spared after excision. Prompt coverage with an FTSG may offer a simple, one-stage low morbidity procedure and early functional recovery $[28,29]$.

\section{Conclusions}

The reviewed literature shows that fingerpad tophi present a difficult diagnosis due to their unusual occurrence and mimicking several other clinical situations [1-6]. Prompt diagnosis and timely initiation of proper therapy with ULT allow one to avoid further complications and surgical interventions may be necessary for early functional recovery $[8,17]$. Such lesions are difficult to remove completely and without sacrificing overlying skin [26]. Thus excision of separable deposits and resurfacing with an FTSG, and simultaneous ULT, may be appropriate treatment for such lesions.

The authors declare no conflict of interest.

\section{References}

1. Shmerling RH, Stern SH, Gravallese EM, Kantrowitz FG. Tophaceous Deposition in the Finger Pads Without Gouty Arthritis. Arch Intern Med 1988; 148: 1830-1832.

2. Braun-Falco M, Hofmann SC.Tophaceous gout in the finger pads. Clin Exp Dermatol 2010; 35: e22-e23.

3. Holland NW, Jost D, Beutler A, et al. Finger Pad Tophi in Gout. J Rheumatol 1996; 23: 690-692.

4. Zarbafian M, Dutz J. Finger pad tophi in a patient with Raynaud phenomenon. JAAD Case Rep 2017; 3: 45-48, DOI: 10.1016/j. jdcr.2016.10.013.

5. Çerman AA, Altunay IK, Salman KE, et al. Finger Pad Tophi in Gout: A Rare Presentation. An Bras Dermatol 2016; 91: 855856, DOI: 10.1590/abd1806-4841.20164466.

6. Chopra KF, Schneiderman P, Grossman ME. Finger pad tophi. Cutis 1999; 64: 233-236.

7. Larmon WA, Kurtz JF. The Surgical Management of Chronic Tophaceous Gout. J Bone Joint Surg Am 1958; 40-A: 743-772.

8. Tripoli M, Falcone AR, Mossuto C, Moschella F. Different Surgical Approaches to Treat Chronic Tophaceous Gout in the Hand: Our Experience. Tech Hand Up Extrem Surg 2010; 14: 187190, DOI: 10.1097/BTH.0b013e3181dcaaa8.

9. Lee $\mathrm{CH}$, Tandon A. Focal hand lesions: review and radiological approach. Insights Imaging 2014; 5: 301-319, DOI: 10.1007/ s13244-014-0334-4.

10. Schuind FA, Stallenberg B, Remmelink M, et al. Associated giant cell tumor and tophaceous deposits in a finger pulp: a case report. J Hand Surg Am 2003; 28: 871-874, DOI: 10.1016/ s0363-5023(03)00255-7.

11. Schumacher HR. Bullous tophi in gout. Ann Rheum Dis 1977; 36: 91-93, DOI: 10.1136/ard.36.1.91.

12. Liu K, Moffatt EJ, Hudson ER, Layfield LJ. Gouty Tophus Presenting as a Soft-Tissue Mass Diagnosed by Fine-Needle Aspiration: A Case Report. Diagn Cytopathol 1996; 15: 246249, DOI: 10.1002/(SICI)1097-0339(199609)15:3<246::AIDDC14>3.0.CO;2-G.

13. Upadhyay N, Saifuddin A. The Radiographic and MRI Features of Gout Referred as Suspected Soft Tissue Sarcoma: A Review of the Literature and Findings From 27 Cases. Skeletal Radiol 2014; 44: 467-476, DOI: 10.1007/s00256-014-2005-4.

14. Popp JD, Bidgood WD Jr, Edwards NL. Magnetic Resonance Imaging of Tophaceous Gout in the Hands and Wrists. Semin Arthritis Rheum 1996; 25: 282-289, DOI: 10.1016/s00490172(96)80038-2.

15. Chou H, Chin TY, Peh WCG. Dual-energy CT in gout - A review of current concepts and applications. I Med Radiat Sci 2017; 64: 41-51, DOI: 10.1002/jmrs.223. 
16. Choi HK, Al-Arfaj AM, Eftekhari A, et al. Dual Energy Computed Tomography in Tophaceous Gout. Ann Rheum Dis 2009; 68 1609-1612, DOI: 10.1136/ard.2008.099713.

17. Kasper IR, Juriga MD, Giurini JM, Shmerling RH. Treatment of Tophaceous Gout: When Medication Is not Enough. Semin Arthritis Rheum 2016; 45: 669-74, DOI: 10.1016/j.semarthrit.2016.01.005.

18. Burns CM, Wortmann RL. Latest evidence on gout management: what the clinician needs to know. Ther Adv Chronic Dis 2012; 3: 271-286, DOI: 10.1177/2040622312462056.

19. Straub LR, Smith JW, Carpenter GK Jr, Dietz G. The Surgery of Gout in the Upper Extremity. JBJS 1961; 43: 731-774.

20.Słowińska I, Słowiński R, Rutkowska-Sak L. Tophi - surgical treatment. Reumatologia 2016; 54: 267-272, DOI: 10.5114/ reum.2016.63819.

21. Meyer Zu Reckendorf G, Dahmam A. Hand involvement in Gout. Hand Surg Rehabil 2018; pii: S2468-1229(18)30065-3, DOI: 10.1016/j.hansur.2018.02.005 [Epub ahead of print].

22. Lee SS, Lin SD, Lai CS, et al. The Soft-Tissue Shaving Procedure for Deformity Management of Chronic Tophaceous Gout. Ann Plast Surg 2003; 51: 372-375, DOI: 10.1097/01. SAP.0000067723.32532.97.
23. Lee SS, Sun IF, Lu YM, et al. Surgical Treatment of the Chronic Tophaceous Deformity in Upper Extremities - The Shaving Technique. J Plast Reconstr Aesthet Surg 2009; 62: 669-674, DOI: 10.1016/j.bjps.2007.12.021.

24. So LWN, Fok MWM, Kwan KYH, et al. Revisiting an Old Surgical Approach to the Management of Tophaceous Gout. Surgical Science 2017; 08: 436-443, DOI: 10.4236/ss.2017.810048.

25. Lui TH. Endoscopic Decompression of a Gouty Tophus at the Hand Dorsum. Arthrosc Tech 2017; 6: e827-e832, DOI: 10.1016/j.eats.2017.02.011.

26. Lee JH, Park JY, Seo JW, et al. Surgical Treatment of Subcutaneous Tophaceous Gout. J Plast Reconstr Aesthet Surg 2010; 63: 1933-1935, DOI: 10.1016/j.bjps.2010.03.019.

27. Kemp TJ, Hirose CB, Coughlin MJ, et al. Treatment of Chronic Tophaceous Gout With a Wound Vacuum-Assisted Device. Foot Ankle Int 2010; 31: 729-731, DOI: 10.3113/FAl.2010.0729.

28. Ertuğrul Sener E, Güzel VB, Takka S. Surgical Management of Tophaceous Gout in the Hand. Arch Orthop Trauma Surg 2000; 120: 482-483, DOI: 10.1007/s004029900061.

29. Lin CT, Chang SC, Chen TM, et al. Free-flap resurfacing of tissue defects in the foot due to large gouty tophi. Microsurgery 2011; 31: 610-615, DOI: 10.1002/micr.20949. 